\title{
Has Ultrasound Elastography come to the age of ripening?
}

\author{
Sorin M. Dudea
}

Radiology Department, "Iuliu Haţieganu” University of Medicine and Pharmacy, Cluj-Napoca, Romania

Definitely, contrast and elastography have been the two words prevailing in the ultrasonography research literature of the last decade. Of these, elastography did cast a special charm over the researchers, due to its ever increasing number of technological approaches. A major role was also played by the adaptability of the method(s) to virtually all applications of ultrasonography and even the opening of novel domains, such as diffuse organ structure assessment. No wonder that dedicated radiologists, mostly breast imaging experts, as well as specialists in gastroenterology, endocrinology, internal medicine etc., were all involved in intensive research and publication.

Truly, elastography boosted ultrasound related publications and rapidly expanded the field of knowledge. Recently, solid publications compacted available knowledge into guidelines that represent a stage benchmark. The most notorious are the EFSUMB guidelines on the clinical use of ultrasound elastography $[1,2]$.

So, after a decade of clinical usage, has elastography come to the age of ripening? The answer to this question requires a graded approach. Overall, the answer is, definitely, no. Or, at least, not in all domains.

Extensive work and widespread experience allow for expert reviews and statements under the form of guidelines for a specific, well documented application of the technique(s). On the other hand, new, potential applications are under permanent scrutiny. Some of the papers

Received Accepted

Med Ultrason

2014, Vol. 16, No 2, 87-88

Corresponding author: Prof. Dr. Sorin M. Dudea Radiology Department Emergency Clinical University Hospital "Iuliu Haţieganu" University of Medicine and Pharmacy, Cluj-Napoca 1-3 Clinicilor str 400006, Cluj-Napoca, Romania e-mail: dudea@clicknet.ro published in this issue of the journal richly substantiate these assertions.

The knowledge gathered by four groups in a specific application of elastography - this being liver disease - allows for the work up of national guidelines and recommendations [3]. This ambitious project is justified both by recent technological developments and by the sizeable number of publications of the authors. Even in this field, serendipitous findings may occur [4].

The applications of elastography in the study of the musculoskeletal system and even more superficial structures, such as skin, are surprisingly sparse. Some of the publications in this issue highlight potential applications, although reported results are, insofar, negative $[5,6]$.

So why is elastography angel and demon? It must relate with the way we understand clinical medicine and imaging. Not only does elastography translate information addressing our least developed sense (tact) into coherent data addressing our most evolved sense (sight), but it also provides figures. Therefore, not only do we need to understand and interpret this "visual tact" and many physical terms we were not aware of but we must also connect this to the figures provided by the machine. It is an angel because it offers figures - a gold mine for statistics. It is a demon because it does not offer figures when we need them or as consistent as we would like them to be. And sometimes the figures are of no use. It is both an angel and a demon because it evolves and new technologies appear, leading to permanent reassessment.

So, returning to the original question:" Has Ultrasound Elastography come to the age of ripening?". In some domains, yes, at least to the extent where it reduced dramatically the number of liver biopsies. In most of the soft tissue applications, no, as newer technologies emerge at a high pace and reassessment is going on continually. Angel or demon, elastography is here to stay and offer us a continual incentive for research. 


\section{References}

1. Bamber J, Cosgrove D, Dietrich CF, et al. EFSUMB guidelines and recommendations on the clinical use of ultrasound elastography. Part 1: Basic principles and technology. Ultraschall Med 2013; 34: 169-184

2. Cosgrove D, Piscaglia F, Bamber J, et al. EFSUMB guidelines and recommendations on the clinical use of ultrasound elastography. Part 2: Clinical applications. Ultraschall Med 2013; 34: 238-253.

3. Sporea I, Bota S, Săftoiu A et al. Romanian National Guidelines and Practical Recommendations on Liver Elastography. Med Ultrason 2014; 16: 123-138.
4. Şirli R, Sporea I, Deleanu A et al. Comparison between the $\mathrm{M}$ and XL probes for liver fibrosis assessment by Transient Elastography. Med Ultrason 2014; 16: 119122.

5. Badea I, Tamas-Szora A, Chiorean I et al. Quantitative assessment of the masseter muscle's elasticity using Acoustic Radiation Force Impulse. Med Ultrason 2014; 16: 8994.

6. Cucoş M, Crişan M, Lenghel M et al. Conventional ultrasonography and sonoelastography in the assessment of plaque psoriasis under topical corticosteroid treatment - work in progress. Med Ultrason 2014; 16: 107113. 